\title{
The Voyager Mission: Encounters With Saturn
}

\author{
E. C. STONE
}

California Institute of Technology

The following reports summarize a number of post-Jupiter aspects of the first phase of the Voyager Mission, a major element in NASA's program of exploration of the outer planets. The objectives of this phase included comparative studies of the Jovian and Saturnian systems and studies of the interplanetary medium at increasing distances from the sun. Scientific reports on the mission through the Jupiter encounters may be found in the Journal of Geophysical Research (86, 8123-8841, 1981). With the completion of the mission through Saturn and with both spacecraft continuing to function well, a second mission phase was added with the objectives of exploring Uranus and investigating the interplanetary medium to beyond $20 \mathrm{AU}$. The Uranus flyby has been chosen so that a third phase can include the exploration of Neptune and extend the investigation of the interplanetary medium to distances beyond $40 \mathrm{AU}$ and at distances of more than $20 \mathrm{AU}$ above the ecliptic.

Because even during the first mission phase the round trip light time of $\sim 3$ hours delayed earth-based reaction to anomalies and restricted real time control of the instruments, the Voyager spacecraft incorporates a number of features that permit autonomous correction for hardware failures and onboard control and execution of complex sequences [Draper et al., 1975]. One computer provides software control of spacecraft attitude and pointing of the scan platform. A second computer controls the scientific instruments and compresses and formats the data for a variety of telemetry modes, while a third computer controls the sequence of scientific and engineering activities.

Among the other important attributes of the spacecraft design is three-axis stabilization, providing long exposure times for imaging of low-contrast objects and long integration times for high-resolution spectrometry. An $X$ band transmitter permitted the return of $\mathbf{4 4 . 8} \mathrm{kbit} / \mathrm{s}$ from Saturn, and with an $S$ band transmitter, provided dual frequency occultation studies of Saturn's rings and of the atmospheres of Saturn and Titan. The spacecraft is powered by radioisotope thermoelectric generators providing $\sim 400 \mathrm{~W}$ of electrical power. An outline of the spacecraft is shown in Figure 1.

In order to address the wide range of scientific objectives of the mission, NASA selected the eleven scientific investigations listed in Table 1. The locations of the instruments are shown in Figure 1, with their nominal characteristics indicated in Table 2. Five of the instruments (the narrow and wide angle cameras, the infrared and ultraviolet spectrometers, and the photopolarimeter) are mounted on the articulated scan platform and are boresighted. More detailed descriptions of the investigations can be found in Space Science Reviews $(21,75$ 376,1977 ), as can a detailed description of the mission design and the selection of the trajectories at Jupiter and Saturn.

Copyright 1983 by the American Geophysical Union.

Paper number 3A1209.

0148-0227/83/003A-1209\$05.00
Voyager 2 was launched from Cape Canaveral, Florida, on August 20, 1977, followed by the launch of Voyager 1 on September 5, 1977. Since Voyager 1 was on a faster trajectory, it encountered Jupiter on March 5, 1979, about four months ahead of Voyager 2, which encountered Jupiter on July 9, 1979. Using the gravity assist of the Jovian flybys, Voyager 1 and Voyager 2 continued their journeys toward Saturn as shown in Figure 2. Voyager 1 swung by Saturn on November 12,1980 , heading up out of the plane of the ecliptic. Voyager 2 followed nine months later, swinging by on August 26, 1981, heading toward an encounter with Uranus in January 1986.

The trajectories of Voyager 1 and Voyager 2 through the Saturnian system were chosen to provide complementary observations. The Voyager 1 trajectory provided a close encounter with Titan, permitting atmospheric occultations, a search for an intrinsic Titanian magnetic field, and studies of the interaction of Titan with either the solar wind or the Saturnian magnetosphere (see Figures 3 and 4). The trajectory was also designed to provide an optimum geometry for the transmission of $S$ and $X$ band radio waves from the spacecraft through the rings, so that the attenuation and scattering of 3.6- and 13-cm waves by the ring particles could be observed. In addition, Voyager 1 provided radio and ultraviolet occultation studies of Saturn's atmosphere as well as close ap-

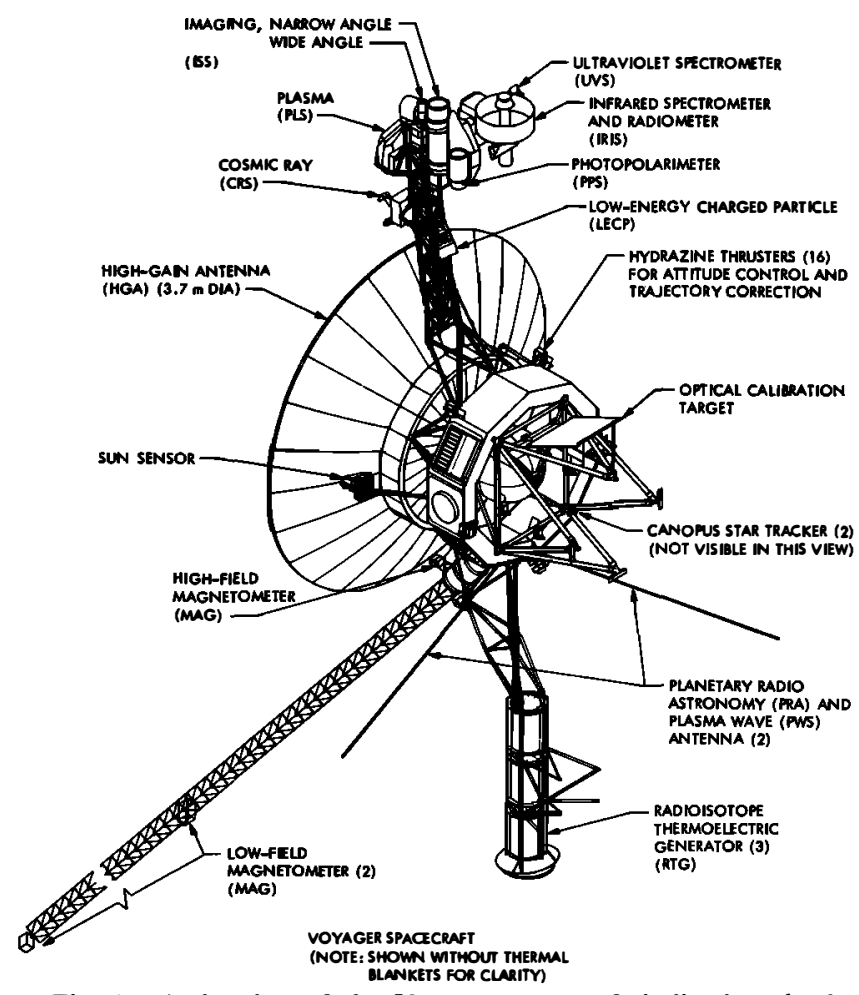

Fig. 1. A drawing of the Voyager spacecraft indicating the location of the science instruments and several spacecraft subsystems. The radio science investigation uses the spacecraft transmitters, an ultrastable oscillator, and the $3.7-\mathrm{m}$ high gain antenna. 
TABLE 1. Voyager Science Investigations

\begin{tabular}{|c|c|}
\hline Investigation Team & $\begin{array}{c}\text { Principal } \\
\text { Investigator/Institution }\end{array}$ \\
\hline Imaging science (ISS) & $\begin{array}{l}\text { Smith/Univ. of Arizona } \\
\text { (Team Leader) }\end{array}$ \\
\hline $\begin{array}{l}\text { Infared spectroscopy and } \\
\text { radiometry (IRIS) }\end{array}$ & Hanel/GSFC \\
\hline Photopolarimetry (PPS) & Lane/JPL \\
\hline Ultraviolet spectroscopy (UVS) & $\begin{array}{l}\text { Broadfoot/Univ. of } \\
\text { Southern Calif. }\end{array}$ \\
\hline Radio science (RSS) & $\begin{array}{l}\text { Tyler/Stanford Univ. } \\
\text { (Team Leader) }\end{array}$ \\
\hline Magnetic fields (MAG) & Ness/GSFC \\
\hline Plasma (PLS) & Bridge/MIT \\
\hline Plasma wave (PWS) & Scarf/TRW \\
\hline Planetary radio astronomy (PRA) & Warwick/Radiophysics, Inc. \\
\hline Low energy charged particles (LECP) & Krimigis/JHU/APL \\
\hline Cosmic rays (CRS) & Vogt/Caltech \\
\hline
\end{tabular}

proaches to three of the moderate-sized icy satellites (Mimas, Dione, and $\mathrm{Rhea}$ ) and to a number of minor satellites.

The much later arrival of Voyager 2 and the Saturn flyby distance were chosen to permit a gravity-assisted continuation on to Uranus and Neptune. However, the exact time of arrival was chosen to provide close approaches to Enceladus and Tethys and closer observations of Hyperion, Iapetus, and Phoebe than achieved by Voyager 1 . The Voyager 2 trajectory also provided closer viewing of the sunlit side of Saturn's rings and permitted measurements in a different region of Saturn's magnetosphere than did Voyager 1. An overview of the Voyager 2 trajectory through the Saturn system is shown in Figures 5 and 6. Table 3 summarizes selected encounter parameters for both spacecraft.

TABLE 2. Typical Instrument Characteristics

\begin{tabular}{|c|c|}
\hline Investigation & Nominal Characteristics \\
\hline ISS & $\begin{array}{l}\text { Two Se-S vidicon cameras ( } f=1500 \mathrm{~mm} \text { and } \\
f=200 \mathrm{~mm}) ; \text { Narrow angle camera; } 19 \mu \mathrm{rad} / \\
\text { line pair, } 2900-6400 \AA \text {. }\end{array}$ \\
\hline IRIS & $\begin{array}{l}\text { Michelson interferometer }(3.3-50 \mu \mathrm{m}) \text { and radi- } \\
\text { ometer }(0.33-2 \mu \mathrm{m}) ; 51 \mathrm{~cm} \text { telescope; } 0 / 25^{\circ} \\
\text { FOV. }\end{array}$ \\
\hline PPS & $\begin{array}{c}\text { Photomultiplier with } 15 \text {-cm telescope; } 2630-7500 \\
\AA ; 3.5^{\circ}, 1^{\circ}, 1 / 4^{\circ}, 1 / 10^{\circ} \mathrm{FOV} ; 2 \text { linear polarizer. }\end{array}$ \\
\hline UVS & $\begin{array}{l}\text { Grating spectrometer; } 500-1700 \AA \text { with } 10-A \text { res- } \\
\text { olution; airglow }\left(1^{\circ} \times 0.1^{\circ} \mathrm{FOV}\right) \text { and occul- } \\
\text { tation }\left(1^{\circ} \times 0.3^{\circ} \mathrm{FOV}\right)\end{array}$ \\
\hline RSS & $\begin{array}{l}\mathrm{S} \text { band }(2.3 \mathrm{GHz}) \text { and } X \text { band }(8.4 \mathrm{GHz}) ; \text { ultra } \\
\text { stable oscillator }\left(<4 \times 10^{-12} \text { short-term drift). }\right.\end{array}$ \\
\hline MAG & $\begin{array}{l}\text { Two low-field }\left(<10^{-6}-0.5 \mathrm{G}\right) \text { and two high-field } \\
\left(5 \times 10^{-4}-20 \mathrm{G}\right) \text { magnetometers; } 13-\mathrm{m} \text { boom; }\end{array}$ \\
\hline & $0-16.7 \mathrm{~Hz}$. \\
\hline PLS & $\begin{array}{l}\text { Earth-pointing sensor (10-eV to 6-keV ions) and } \\
\text { lateral sensor (10-eV to 6-keV ions, 4-eV to } \\
\text { 6-keV electrons). }\end{array}$ \\
\hline PWS & $\begin{array}{l}\text { Sixteen channels }(10 \mathrm{~Hz}-56.2 \mathrm{kHz}) \text {; waveform } \\
\text { analyzer }(150 \mathrm{~Hz}-10 \mathrm{kHz}) \text {; share PRA an- } \\
\text { tennas. }\end{array}$ \\
\hline PRA & $\begin{array}{l}\text { Stepping receiver ( } 1.2 \mathrm{kHz} \text { and } 20.4 \mathrm{kHz}-40.5 \\
\mathrm{MHz}) ; \text { right and left circular polarization; or- } \\
\text { thogonal } 10 \mathrm{~m} \text { monopole antennas. }\end{array}$ \\
\hline LECP & $\begin{array}{l}\text { Two solid-state detector systems on rotating plat- } \\
\text { form; } 10 \mathrm{keV}-10 \mathrm{MeV} \text { electrons; } 10 \\
\mathrm{keV} / \text { nucleon-150 MeV/nucleon ions. }\end{array}$ \\
\hline CRS & $\begin{array}{l}\text { Multiple solid-state detector telescopes; 3-110 } \\
\text { MeV electrons; 1-500 MeV/nucleon nuclei; } \\
\text { three-dimensional anisotropies. }\end{array}$ \\
\hline
\end{tabular}

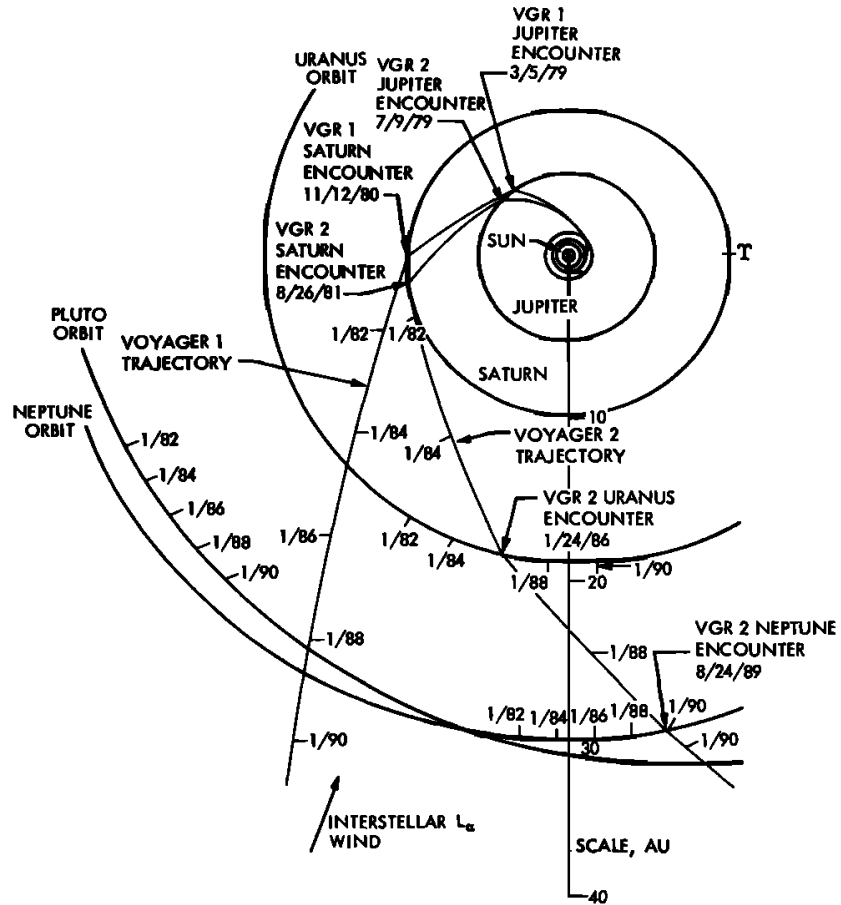

Fig. 2. A view normal to the ecliptic plane of the Voyager 1 and 2 trajectories.

The eleven science teams undertook a wide range of studies of the Saturnian system, including studies of the planet, the rings, the satellites, the magnetosphere, and the interplanetary medium between Jupiter and Saturn. Planning for these studies benefited significantly from continuing earth-based studies and from the Pioneer 11 flyby studies as reported in Science $(207,400-453,1980)$ and in the Journal of Geophysical Re-

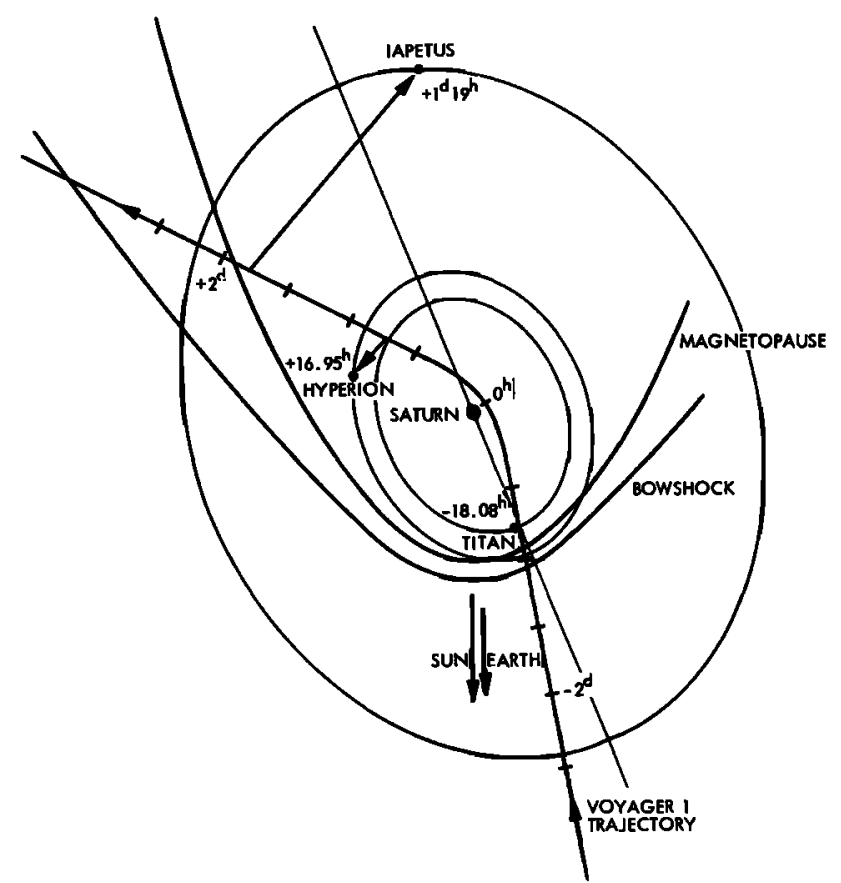

Fig. 3. A view normal to the trajectory plane of the Voyager 2 path through the Saturn system. The projected orbits of three outer satellites are shown with Iapetus orbiting Saturn at a distance of $\sim 59$ $\boldsymbol{R}_{\boldsymbol{s}}$. An arrow connects the location of the spacecraft and each satellite at the time of closest approach to that satellite by Voyager 1. Time ticks along the trajectory indicate 12-hour intervals. 


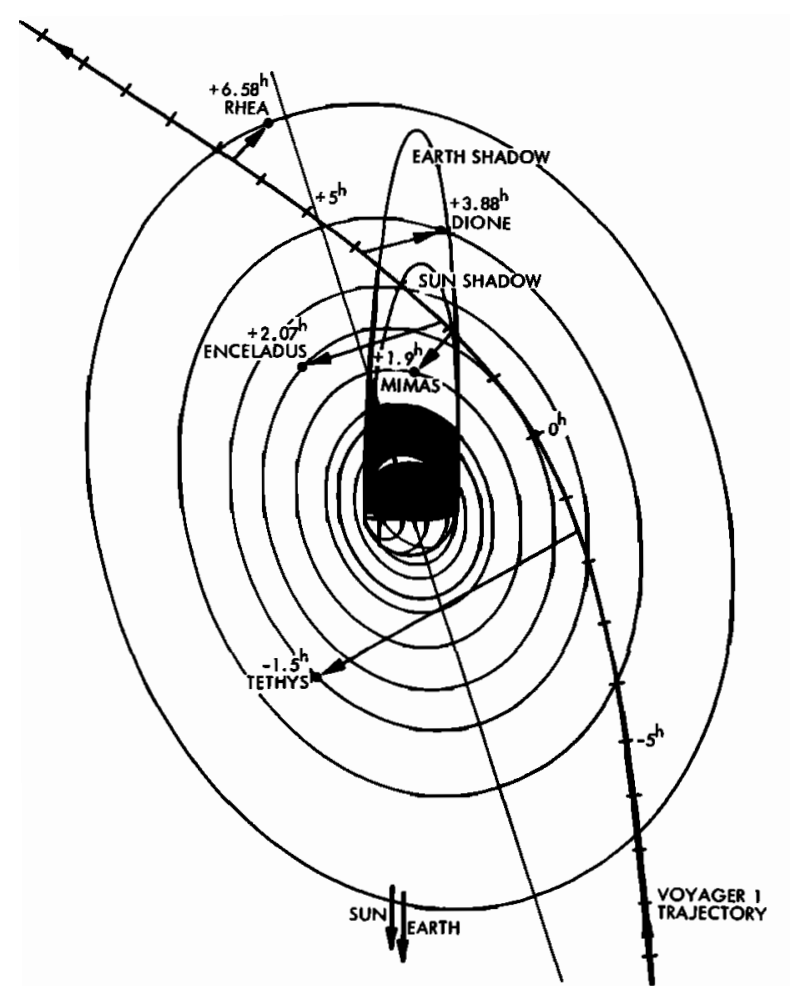

Fig. 4. A view similar to Figure 3 of the Voyager 1 path through the orbits of the inner satellites (Rhea orbits at $\sim 8.7 R_{s}$ ). Time ticks indicate 1-hour intervals.

search $(85,5651-5956,1980)$. The initial reports of the Voyager 1 encounter results were published in Science $(212,159-$ $243,1981)$, and in Nature $(292,675-755,1981)$, followed by the initial Voyager 2 reports in Science $(215,499-594,1982)$. A number of more detailed reports have subsequently appeared, including papers in two special Saturn issues of Icarus (53

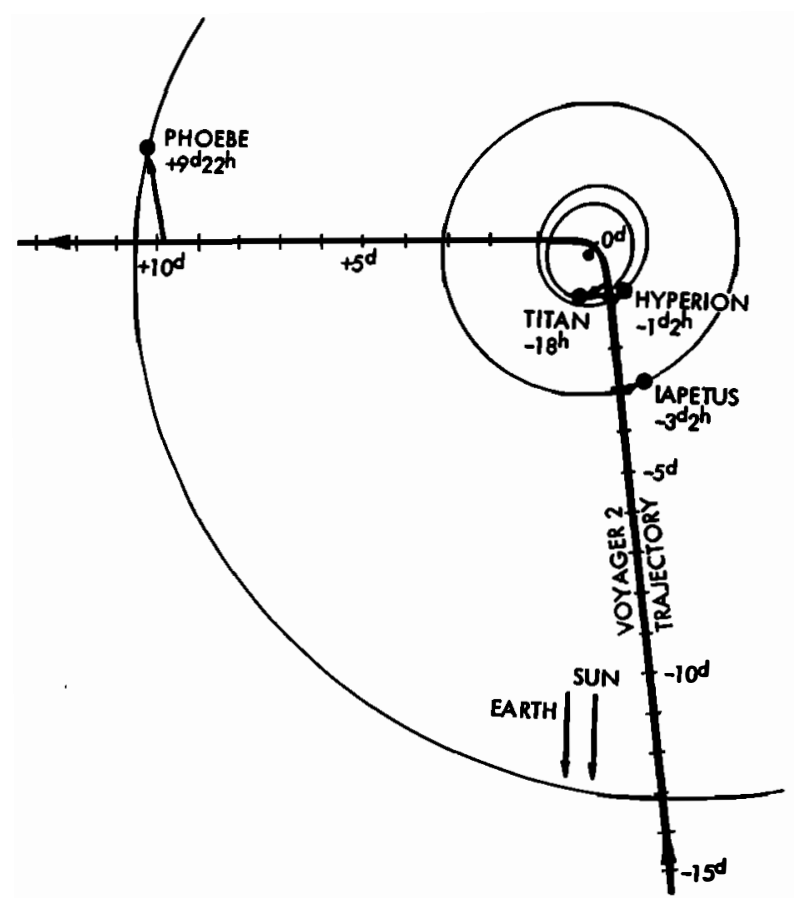

Fig. 5. A view similar to Figure 3 of the Voyager 2 path through the Saturn system. Phoebe orbits at $\sim 215 R_{s}$. Time ticks indicate 1-day intervals.

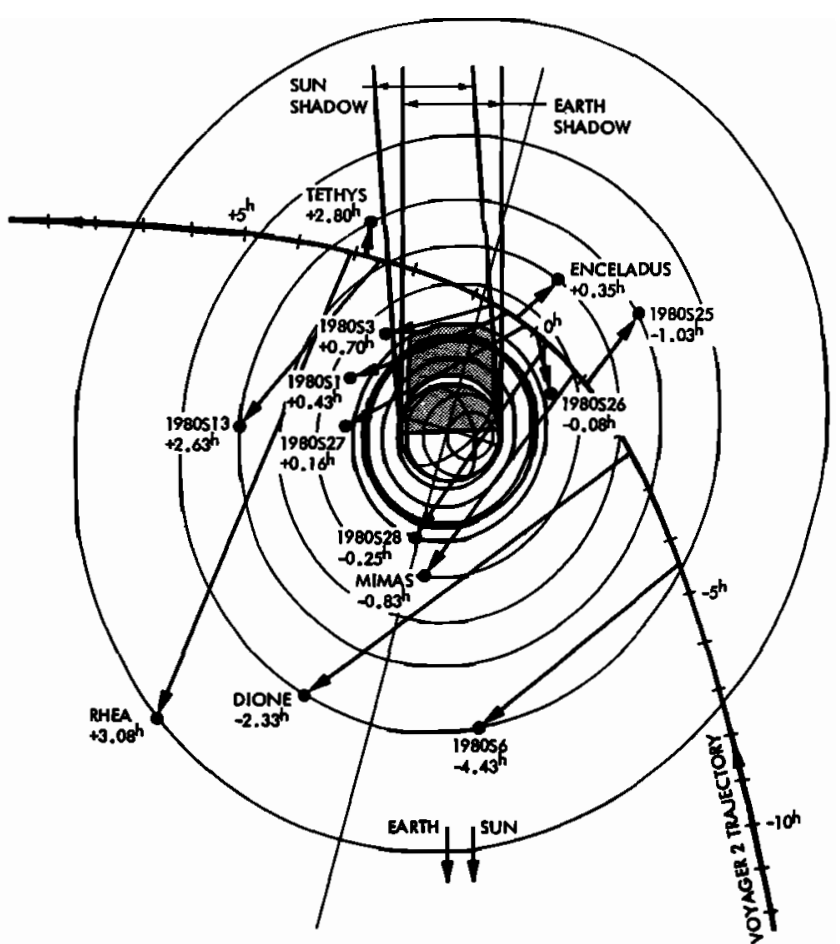

Fig. 6. A view similar to Figure 3 of the Voyager 2 path through the orbits of the inner satellites (Rhea orbits at $\sim 8.7 R_{S}$ ). Time ticks indicate 1-hour intervals.

163-387, 1983, and 54,160-360, 1983) and a collection of papers on Titan in the Journal of Geophysical Research (87, 1351-1418, 1982).

All of the data from the first mission phase will continue to be a significant resource in the detailed studies of the giant planets, their rings, satellites, and magnetospheres, and the intervening interplanetary medium. Both Voyagers, in conjunction with Pioneer 10 and 11 (see, for example, Northrop et al., [1980]), will continue to explore new regions of the heliosphere, with the possibility of eventually observing the heliospheric boundary. In 1990, Voyager 1 will be at $40 \mathrm{AU}$,

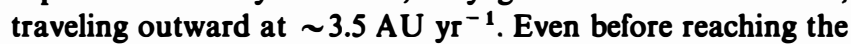
boundary, the spacecraft may detect cosmic rays from nearby

TABLE 3. Selected Voyager Encounter Parameters

\begin{tabular}{lrrr}
\hline & \multicolumn{3}{c}{ Distance, km } \\
\cline { 2 - 4 } \multicolumn{1}{c}{ Satellite } & Voyager 1 & Voyager 2 & \multicolumn{1}{c}{ Saturn * } \\
\hline Atlas & 219,000 & 287,000 & 137,670 \\
1980S27 & 300,000 & 247,000 & 139,350 \\
1980S26 & 270,000 & 107,000 & 141,700 \\
Epimetheus & 121,000 & 147,000 & 151,422 \\
Janus & 297,000 & 223,000 & 151,472 \\
Mimas & 88,440 & 309,930 & 185,540 \\
Enceladus & 202,040 & 87,010 & 238,040 \\
Tethys & 415,670 & 93,010 & 294,670 \\
1980S6 & 230,000 & 270,000 & 378,060 \\
Dione & 161,520 & 502,310 & 377,420 \\
Rhea & 73,980 & 645,260 & 527,100 \\
Titan & 6,490 & 666,190 & $1,221,860$ \\
Hyperion & 880,440 & 471,370 & $1,481,000$ \\
Iapetus & $2,470,000$ & 908,680 & $3,560,800$ \\
Phoebe & $13,500,000$ & $2,075,640$ & $12,954,000$ \\
\hline
\end{tabular}

*Distance to center of planet. Saturn's equatorial radius is 60,330 $\mathrm{km}$. 
regions of our galaxy, which are of such low energy that the radially streaming solar wind limits their penetration to the - outer fringes of the heliosphere.

During this same period, Voyager 2 will be encountering Uranus and Neptune about which much less is known than was known about Jupiter and Saturn prior to the Voyager encounters. Thus further discoveries await the continuing exploration of the outer solar system.

Acknowledgments. The Voyager Project is managed by the Jet Propulsion Laboratory of the California Institute of Technology under NASA contract NAS7-918 (formerly NAS7-100). The following individuals coauthored the initial Saturn reports in Science $(212,159$ 243, 1981), with the additional authors indicated for Voyager 2 (Science, 215, 499-594, 1982):

ISS: B. A. Smith, L. Soderblom, R. Beebe, J. Boyce, G. Briggs, A. Bunker, S. A. Collins, C. J. Hansen, T. V. Johnson, J. L. Mitchell, R. J. Terrile, M. Carr, A. F. Cook II, J. Cuzzi, J. B. Pollack, G. E. Danielson, A. Ingersoll, M. E. Davies, G. E. Hunt, H. Masursky, E. Shoemaker, D. Morrison, T. Owen, C. Sagan, J. Veverka, R. Strom, V. E. Suomi; Orbits: S. P. Synnott, C. F. Peters, B. A. Smith, L. A. Morabito; Voyager 2: R. Batson, P. Bridges, J. Inge.

IRIS: R. Hanel, B. Conrath, F. M. Flasar, V. Kunde, W. Maguire, J. Pearl, J. Pirraglia, R. Samuelson, L. Herath, H. Allison, D. Cruikshank, D. Gautier, P. Gierasch, L. Horn, R. Koppany, D. Ponnamperuma.

PPS: A. L. Lane, C. W. Hord, R. A. West, L. W. Esposito, D. L. Coffeen, M. Sato, K. E. Simmons, R. B. Pomphrey, R. B. Morris.

UVS: A. L. Broadfoot, B. R. Sandel, D. E. Shemansky, J. B. Holberg, G. R. Smith, D. F. Strobel, J. C. McConnell, S. Kumar, D. M. Hunten, S. K. Atreya, T. M. Donahue, H. W. Moos, J. L. Bertaux, J. E. Blamont, R. B. Pomphrey, S. Linick.
RSS: G. L. Tyler, V. R. Eshleman, J. D. Anderson, G. S. Levy, G. F. Lindal, G. E. Wood, T. A. Croft.

MAG: N. F. Ness, M. H. Acuna, R. P. Lepping, J. E. P. Connerney, K. W. Behannon, L. F. Burlaga, F. M. Neubauer.

PLS: H. S. Bridge, J. W. Belcher, A. J. Lazarus, S. Olbert, J. D. Sullivan, F. Bagenal, P. R. Gazis, R. E. Hartle, K. W. Ogilvie, J. D. Scudder, E. C. Sittler, A. Eviatar, G. L. Siscoe, C. K. Goertz, V. M. Vasyliunas; Voyager 2: R. L. McNutt.

PWS: D. A. Gurnett, W. S. Kurth, F. L. Scarf; Voyager 2: R. L. Poynter.

PRA: J. W. Warwick, J. B. Pearce, D. R. Evans, T. D. Carr, J. J. Schauble, J. K. Alexander, M. L. Kaiser, M. D. Desch, B. M. Pedersen, A. Lecacheux, G. Daigne, A. Boischot, C. H. Barrow; Voyager 2: J. H. Romig, M. Aubier, Y. Leblanc.

LECP: S. M. Krimigis, T. P. Armstrong, W. I. Axford, C. O. Bostrom, G. Gloeckler, E. P. Keath, L. J. Lanzerotti, J. F. Carbary, D. C. Hamilton, E. C. Roelof.

CRS: R. E. Vogt, D. L. Chenette, A. C. Cummings, T. L. Garrard, E. C. Stone, A. W. Schardt, J. H. Trainor, N. Lal, F. B. McDonald.

\section{REFERENCDS}

Draper, R. F., W. I. Prudy, and G. Cunningham, The outer planet mariner spacecraft, paper presented at the AIAA/AGU Conference on the Exploration of the Outer Planets, St. Louis, Mo., 1975.

Northrop, T. G., A. G. Opp, and J. H. Wolfe, Pioneer 11 Saturn encounter, J. Geophys. Res., 85, 5651, 1980.

E. C. Stone, 103-33 E. Bridge Laboratory, California Institute of Technology, Pasadena, CA 91125.

(Received June 17, 1983;

revised July 22, 1983;

accepted July 22, 1983.) 


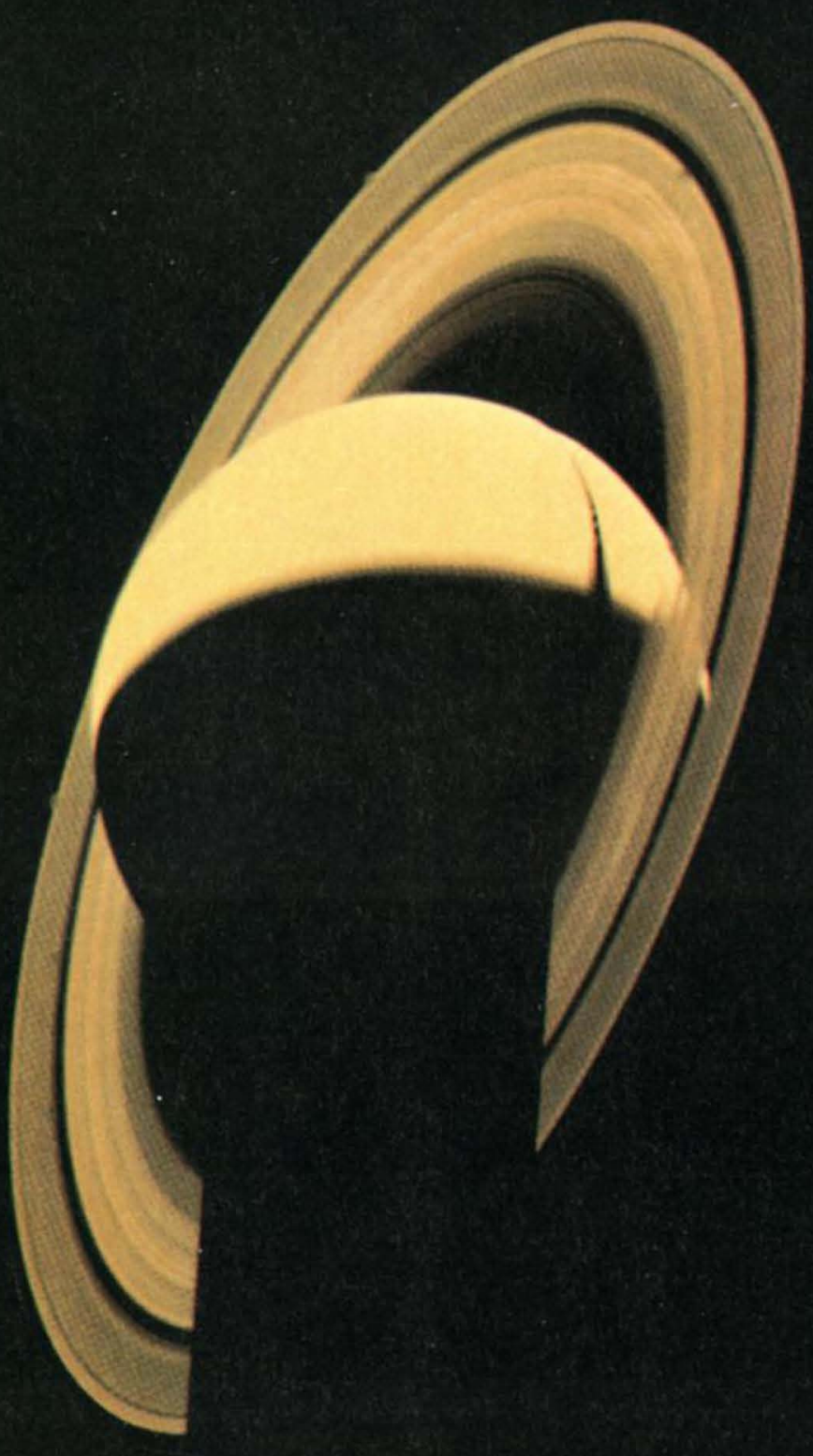

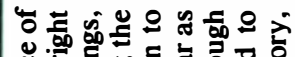

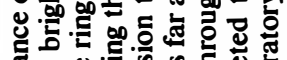

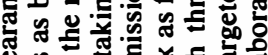
品

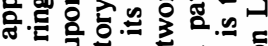
o

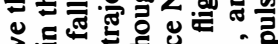

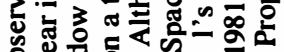
०० 응

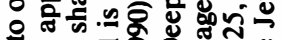
\%

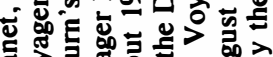
สํㅇำ \% का न 合它 के E

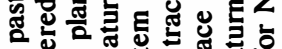

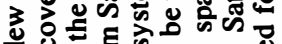

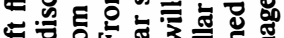
割

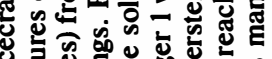

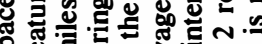
क人

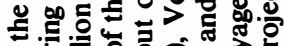
乌 흘

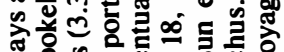

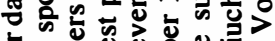

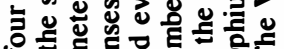

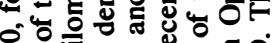
\& 远 살

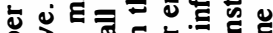

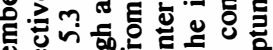

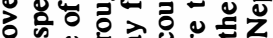
Z ᄃ 뭉 E.w.

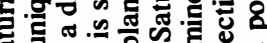
का ส

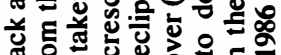
인.

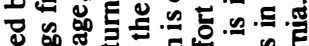

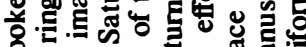

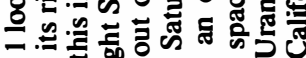

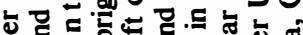

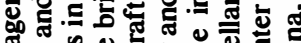

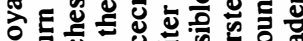
งทำ 